\title{
Dietary intakes of fats, fish and nuts and olfactory impairment in older adults
}

\author{
Bamini Gopinath $^{1 *}$, Carolyn M. Sue ${ }^{2}$, Victoria M. Flood ${ }^{3,4}$, George Burlutsky $^{1}$ and Paul Mitchell ${ }^{1}$ \\ ${ }^{1}$ Centre for Vision Research, Department of Ophthalmology and Westmead Millennium Institute, University of Sydney, \\ Sydney, NSW 2145, Australia \\ ${ }^{2}$ Departments of Neurology and Neurogenetics, Kolling Institute, University of Sydney, Sydney, NSW, Australia \\ ${ }^{3}$ Faculty of Health Sciences, University of Sydney, Sydney, NSW, Australia \\ ${ }^{4}$ St Vincent's Hospital, Sydney, NSW, Australia \\ (Submitted 14 December 2014 - Final revision received 23 February 2015 - Accepted 23 March 2015 - First published online 16 June 2015)
}

\section{Abstract}

It is unclear whether lifestyle modifications, such as dietary changes, should be advocated to prevent olfactory dysfunction. We investigated the association between dietary intakes of fats (saturated, mono-unsaturated and polyunsaturated fats, and cholesterol) and related food groups (nuts, fish, butter, margarine) with olfactory impairment. There were 1331 and 667 participants (older than 60 years) at baseline and 5-year follow-up, respectively, with complete olfaction and dietary data. Dietary data were collected using a validated semi-quantitative FFQ. Olfaction was measured using the San Diego Odor Identification Test. In a cross-sectional analysis of baseline data, those in the highest $v$. lowest quartile of $n$-6 PUFA intake had reduced odds of having any olfactory impairment, multivariable-adjusted OR 0.66 ( $95 \%$ CI 0.44, 0.97), $P$ for trend $=0.06$. Participants in the highest $v$. lowest quartile of margarine consumption had a $65 \%$ reduced odds of having moderate/severe olfactory impairment ( $P$ for trend $=0.02$ ). Participants in the highest quartile compared to the lowest quartile (reference) of nut consumption had a $46 \%$ ( $P$ for trend $=0.01)$ and $58 \%(P$ for trend $=0.001)$ reduced odds of having any or mild olfactory impairment, respectively. Older adults in the highest $v$. lowest quartile of fish consumption had $35 \%$ ( $P$ for trend $=0.03)$ and $50 \%(P$ for trend $=0.01)$ reduced likelihood of having any or mild olfactory impairment, respectively. In longitudinal analyses, a marginally significant association was observed between nut consumption and incidence of any olfactory impairment, highest $v$. lowest quartile of nut consumption: OR $0 \cdot 61$ (95\% CI $0 \cdot 37,1 \cdot 00)$. Older adults with the highest consumption of nuts and fish had reduced odds of olfactory impairment, independent of potential confounding variables.

\section{Key words: Fatty acids: Nuts: Fish: Olfactory impairment: Blue Mountains Eye Study}

A decrease in olfactory function with increasing age has been extensively reported ${ }^{(1-3)}$. Unlike alterations in hearing and vision, age-related changes in olfaction often go unnoticed, and smell ability is rarely evaluated clinically ${ }^{(4)}$. There are several bases for age-related changes in smell function and include some of the following: (1) ossification and closure of the foramina of the cribriform plate ${ }^{(5)}$; (2) development of early neurodegenerative disease pathology ${ }^{(6)}$; and (3) cumulative damage to the olfactory receptors from smoking and other insults throughout life ${ }^{(1,4)}$. There have been various tests developed to evaluate the sense of smell. Included in such procedures are tests of olfactory sensitivity (e.g. odour detection and recognition thresholds), odour discrimination, odour identification, odour memory and supra-threshold scaling of odour intensities and pleasantness ${ }^{(7)}$.

We previously showed that among the Blue Mountains Eye Study (BMES) participants aged $\geq 60$ years, the prevalence of olfactory impairment was $27 \cdot 0 \%{ }^{(2)}$. This was comparable to the $24.5 \%$ rate observed in the Wisconsin Epidemiology of Hearing Loss Study for persons aged 43-86 years ${ }^{(3)}$. In addition to its relatively high prevalence, olfactory loss impacts on a wide range of functions. Decreased olfaction results in reductions in appetite, leading to weight loss and malnutrition $^{(8)}$. In the presence of impaired smell, disability and diminished quality of life are observed ${ }^{(9-11)}$. Many studies have also shown a significant relationship between olfactory impairment and depressive symptoms and poor quality of life ${ }^{(11,12)}$. In addition, the functional ability and independence of older adults is significantly impaired in the presence of olfactory impairment ${ }^{(1)}$. More recently, olfactory impairment has been shown to predict future cognitive decline ${ }^{(13)}$, Parkinson's disease ${ }^{(14)}$ and increased mortality risk ${ }^{(15)}$.

Apart from the effects of smoking, there are very few epidemiological data on modifiable risk factors associated with

Abbreviations: BMES, Blue Mountains Eye Study; SDOIT, San Diego Odor Identification Test.

*Corresponding author: B. Gopinath, fax +612 86273099, email bamini.gopinath@sydney.edu.au 
olfactory impairment. A better understanding of lifestyle risk factors will facilitate to identify and change at-risk behaviour, and help in implementing preventive strategies. Encouraging changes in the dietary intakes of individuals could be a potential preventive strategy for olfactory impairment. A recent animal study by Thiebaud et al. ${ }^{(16)}$ showed that exposure to a high-fat diet, independent of the degree of adiposity, can impact on the general neuro-architecture of the olfactory system. Specifically, mice fed a high-fat diet showed a marked reduction in olfactory discrimination. The authors speculated that such reduction in sensory performance, which is precipitated by a chronic increase in fat in the diet, could be linked to the observed loss of olfactory sensory neurons in the study or just be one facet of the likely multiple changes in the different levels of the olfactory system attributed to the presence of fat ${ }^{(16)}$. However, to our best knowledge, no adult population-based study has specifically examined the association between dietary intakes of fats or the major fat-containing groups and the prevalence and incidence of olfactory impairment.

We therefore used a representative community-based cohort of older adults to investigate the following aims: (1) to establish whether cross-sectional and longitudinal associations exist between dietary fats (saturated, monounsaturated, polyunsaturated, cholesterol), certain food groups (e.g. nuts, butter, margarine and fish) and olfactory loss; and (2) to determine whether observed cross-sectional or longitudinal associations differed according to the severity of olfactory impairment.

\section{Methods}

\section{Study population}

The BMES is a population-based cohort study of common eye diseases and other health outcomes in a suburban Australian population located west of Sydney. Study methods and procedures have been described elsewhere ${ }^{(17)}$. Baseline examinations of 3654 residents aged $>49$ years were conducted during 1992-4 (BMES-1, 82.4\% participation rate). Surviving baseline participants were invited to attend examinations after 5 years (1997-9, BMES-2), 10 years (2002-4, BMES-3) and 15 years (2007-9, BMES-4). At BMES-2, -3 and -4, 2334 participants $(75.1 \%$ of survivors), 1952 participants $(75.6 \%$ of survivors) and 1149 (55.4\% of survivors) with complete data were re-examined, respectively. The present study was conducted according to the Declaration of Helsinki and The University of Sydney and the Western Sydney Area Human Ethics Committees approved the study, and written, informed consent was obtained from all participants at each examination.

\section{Olfaction examination}

The San Diego Odor Identification Test (SDOIT) ${ }^{(18)}$ and related olfactory questions were a component of the BMES-3 examination, and complete olfaction data were obtained from 1636 of 1952 (83.8\%) BMES-3 participants. Participants were tested individually with the SDOIT, an eight-item odour identification test with a test-retest reliability relatively similar to that for the forty-item University of Pennsylvania Smell Identification Test (UPSIT) $\left(r \begin{array}{lllll} & 0.86 & \text { SDOIT; } & r & 0.94\end{array}\right.$ UPSIT $^{(19)}$. Odorants were presented to the participants in a random order, in an opaque container covered with gauze. These odorants are common odours in the household, that is, chocolate, cinnamon, talcum powder, chewing gum, coffee, mustard, peanut butter, and play-doh. An interstimulus pause of $45 \mathrm{~s}$ was used to prevent adaptation ${ }^{(20)}$. The examiner would record on a score sheet ' 0 ' (incorrect), '1' (correct) or '9' (don't know) beside each listed odorant. A picture board illustrating the odorants as well as distracters was used for participants to identify each odorant. Scores were calculated from the number of odorants identified correctly. We defined mild olfactory impairment as less than six but greater than three correct responses and moderate/ severe as three or less correct responses out of a total of eight possible responses.

\section{Dietary assessment}

Dietary data were collected using a 145-item self-administered FFQ, modified for Australian diet and vernacular from an early Willett $\mathrm{FFQ}^{(21)}$, and included reference portion sizes. Participants used a nine-category frequency scale to indicate the usual frequency of consuming individual food items during the past year ${ }^{(22)}$. The FFQ showed moderate to good agreement for ranking individuals according to their fat intakes (total fat $r$ 0.68, SFA $r$ 0.67, MUFA $r$ 0.54, and PUFA $r 0.44$ ) and correctly classified more than $70 \%$ of people within one quintile for all types of fat ${ }^{(22,23)}$. Total dietary fat comprised dietary cholesterol, SFA, MUFA and PUFA.

Dietary intakes were estimated using the Australian Tables of Food Composition (NUTTAB95) ${ }^{(23)}$. Additional fatty acid food composition data were added from the Royal Melbourne Institute of Technology database ${ }^{(24)}$, available on FoodWorks, version 3 (Xyris Software Private Limited). Dietary fat intakes were expressed as a contribution to percentage of energy intake. Long-chain $n$-3 PUFA was calculated from the sum of EPA (20:5n-3), docosapentaenoic (22:5n-3) and DHA (22:6n-3). Total $n-3$ PUFA consumption was calculated by adding the intakes of long-chain $n$ - 3 PUFA and $\alpha$-linolenic acid. All dietary sources of $n-3$ PUFA were included in the analyses. In previously published fatty acid food sources of this population $^{(25)}$, we have described the various food sources, for example, for total $n$-3 PUFA intake the main dietary sources were from fats and oils (27\%), fish and seafood (21\%), meat and meat products (13\%), breads and cereals (8\%), milk and milk products (8\%); the main food sources of long-chain $n$-3 PUFA were fish and seafood (69\%), meat and meat products (15\%), and eggs (15\%; includes $n$ - 3 fortified eggs). The FFQ includes specific questions about the type of oil and margarine, which were individually coded and included in the total PUFA analyses. This analysis of the baseline dietary intake does not include $n$ - 3 from supplements. Previous investigations have indicated supplement use of $n$-3/fish oils was not high in this cohort ${ }^{(26)}$. The total 


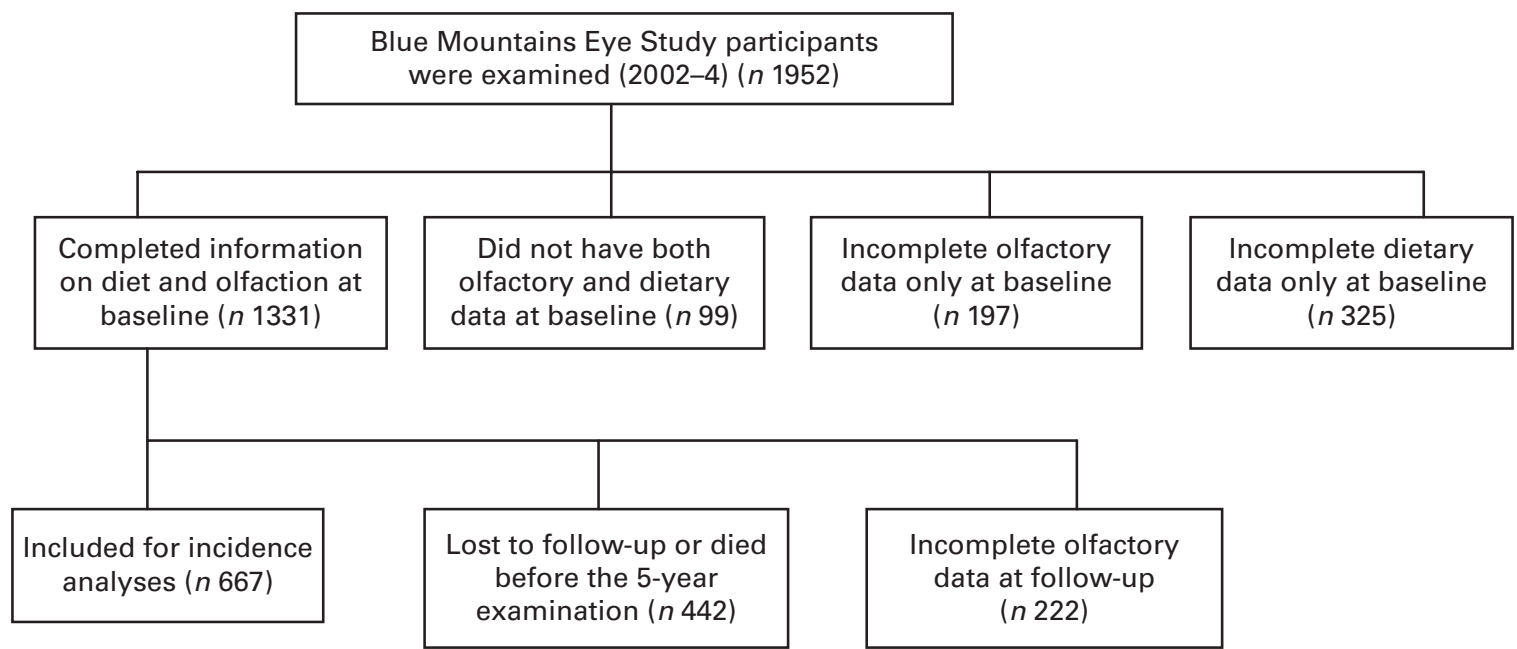

Fig. 1. Flowchart of study participation in the Blue Mountains Eye Study from 2002-4 to 2007-9.

$n$-6 PUFA consumption was calculated by adding the intakes of linoleic and arachidonic $\operatorname{acid}^{(27)}$.

\section{Assessment of covariates}

At face-to-face interviews with trained interviewers, a comprehensive medical history that included information about hearing, demographic factors, socio-economic characteristics and lifestyle factors was obtained from all participants.
Smoking status was determined from history as never smoked, past smoker and current smoker (which included those who had ceased smoking within the past 12 months). Information on all currently taken medications was considered to determine the use of cholesterol-lowering medication (i.e. statins). Diabetes was defined either by history or from fasting blood glucose $\geq 7.0 \mathrm{mmol} / 1$. Subjects were defined as having hypertension if they had systolic blood pressure greater than $140 \mathrm{mmHg}$ or diastolic blood pressure more than $90 \mathrm{mmHg}$

Table 1. Study characteristics of Blue Mountains Eye Study participants by presence of olfactory impairment (Mean values and standard deviations; number of participants and percentages)

\begin{tabular}{|c|c|c|c|c|c|}
\hline \multirow[b]{3}{*}{ Characteristics } & \multicolumn{4}{|c|}{ Participants ( $n$ 1331) } & \multirow[b]{3}{*}{$P$} \\
\hline & \multicolumn{2}{|c|}{$\begin{array}{c}\text { No olfactory } \\
\text { impairment }(n \text { 1013) }\end{array}$} & \multicolumn{2}{|c|}{$\begin{array}{l}\text { Olfactory impairment } \\
\qquad(n 318)\end{array}$} & \\
\hline & Mean & SD & Mean & SD & \\
\hline Age (years) & 71.9 & $7 \cdot 1$ & $76 \cdot 4$ & $7 \cdot 2$ & $<0.0001$ \\
\hline Men & & & & & $<0.0001$ \\
\hline$n$ & \multicolumn{2}{|c|}{377} & \multicolumn{2}{|c|}{176} & \\
\hline$\%$ & \multicolumn{2}{|c|}{$37 \cdot 2$} & \multicolumn{2}{|c|}{$55 \cdot 4$} & \\
\hline $\mathrm{BMI}\left(\mathrm{kg} / \mathrm{m}^{2}\right)$ & $27 \cdot 6$ & 4.6 & $26 \cdot 0$ & $4 \cdot 2$ & $<0.0001$ \\
\hline Current smoking & & & & & 0.23 \\
\hline$n$ & \multicolumn{2}{|c|}{66} & \multicolumn{2}{|c|}{27} & \\
\hline$\%$ & \multicolumn{2}{|c|}{6.5} & \multicolumn{2}{|c|}{8.5} & \\
\hline History of hypertension & & & & & 0.88 \\
\hline$n$ & \multicolumn{2}{|c|}{579} & \multicolumn{2}{|c|}{180} & \\
\hline$\%$ & \multicolumn{2}{|c|}{$57 \cdot 4$} & \multicolumn{2}{|c|}{$57 \cdot 0$} & \\
\hline History of diabetes & & & & & $0 \cdot 88$ \\
\hline$n$ & \multicolumn{2}{|c|}{579} & \multirow{2}{*}{\multicolumn{2}{|c|}{$\begin{array}{c}180 \\
57.0\end{array}$}} & \\
\hline$\%$ & \multicolumn{2}{|c|}{57.4} & & & \\
\hline Total energy intake $(\mathrm{kJ} / \mathrm{d})$ & $8668 \cdot 2$ & 2469 & $8781 \cdot 2$ & 2503 & 0.48 \\
\hline Total dietary fat intake $(\mathrm{g} / \mathrm{d})$ & $76 \cdot 1$ & $28 \cdot 1$ & $76 \cdot 7$ & 28.3 & 0.72 \\
\hline Saturated fat intake $(\mathrm{g} / \mathrm{d})$ & $29 \cdot 3$ & $12 \cdot 4$ & 30.5 & 13.5 & $0 \cdot 12$ \\
\hline Monounsaturated fat intake $(\mathrm{g} / \mathrm{d})$ & $27 \cdot 8$ & $10 \cdot 6$ & $27 \cdot 7$ & $10 \cdot 4$ & 0.88 \\
\hline$n-3$ PUFA intake (g/d) & $1 \cdot 1$ & 0.6 & 1.1 & 0.6 & 0.27 \\
\hline$n-6$ PUFA intake $(\mathrm{g} / \mathrm{d})$ & $9 \cdot 1$ & $4 \cdot 7$ & $8 \cdot 6$ & 4.4 & 0.09 \\
\hline Cholesterol intake $(\mathrm{g} / \mathrm{d})$ & 314.1 & $146 \cdot 3$ & $317 \cdot 8$ & $150 \cdot 3$ & 0.70 \\
\hline Nuts $(g / d)$ & $9 \cdot 7$ & $14 \cdot 3$ & $7 \cdot 8$ & $12 \cdot 4$ & 0.02 \\
\hline Margarine $(g / d)$ & $10 \cdot 2$ & $9 \cdot 3$ & $10 \cdot 3$ & $9 \cdot 1$ & 0.94 \\
\hline Butter $(\mathrm{g} / \mathrm{d})$ & $3 \cdot 6$ & 6.5 & 3.5 & $7 \cdot 0$ & 0.98 \\
\hline Fish $(\mathrm{g} / \mathrm{d})$ & 39.4 & 35.4 & 34.4 & $32 \cdot 9$ & 0.03 \\
\hline
\end{tabular}


or were on anti-hypertensive medications ${ }^{(28)}$. BMI was calculated as weight divided by height squared $\left(\mathrm{kg} / \mathrm{m}^{2}\right)$.

\section{Results}

Of the 1952 participants examined at BMES-3 (or baseline for the purpose of the present study), 1331 had complete information on dietary intakes and olfactory status (Fig. 1). Study participants with olfactory impairment compared to those with normal olfaction were more likely to be male, older and have a lower BMI and lower consumption of fish and nuts (Table 1). After multivariable adjustment, a marginally significant association was observed between increasing dietary intakes of saturated fats and increased odds of prevalent mild olfactory impairment, $P$ for trend $=0.05$ (Table 2). Participants in the highest compared to lowest quartile of total $n-6$ PUFA had a $34 \%$ reduced odds of having any olfactory impairment ( $P$ for trend=0.06). Non-significant associations were observed between dietary intakes of other types of fats and prevalence of olfactory impairment (Table 2).
Participants in the highest quartile compared to the lowest quartile of nut consumption had a $46 \%$ ( $P$ for trend $=0 \cdot 01$ ) and $58 \%$ ( $P$ for trend=0.001) reduced odds of having any or mild prevalent olfactory impairment, respectively (Table 3). In addition, participants in the highest $v$. lowest quartile of margarine consumption had a $65 \%$ reduced odds of having moderate/severe olfactory impairment ( $P$ for trend $=0.02)$. Participants who were in the highest compared to the lowest quartile of fish consumption had a 35\% ( $P$ for trend $=0.03$ ) and 50\% ( $P$ for trend $=0.01)$ reduced likelihood of having any or mild olfactory impairment (Table 3 ).

Of the 1331 participants included for cross-sectional analyses, 667 participants had complete olfactory function data at the 5-year follow-up (Fig. 1). Of these, 174 (26\%) developed incident (i.e. new cases) of any olfactory impairment. Table 4 shows a marginally significant association between nut consumption, and 5-year incidence of any olfactory impairment was observed, that is, those in the highest $v$. lowest quartile of nut consumption at baseline had reduced risk of developing incident olfactory impairment, multivariable-adjusted OR

Table 2. Association between dietary fats and prevalence of olfactory loss in the Blue Mountains Eye Study (Adjusted odds ratios and $95 \%$ confidence intervals, $n$ 1331)

\begin{tabular}{|c|c|c|c|c|c|c|}
\hline \multirow[b]{3}{*}{ Variable } & \multicolumn{6}{|c|}{ Severity of olfactory impairment ${ }^{\star}$} \\
\hline & \multicolumn{2}{|c|}{ Any } & \multicolumn{2}{|r|}{ Mild } & \multicolumn{2}{|c|}{ Moderate/severe } \\
\hline & OR & $95 \% \mathrm{Cl}$ & OR & $95 \% \mathrm{Cl}$ & OR & $95 \% \mathrm{Cl}$ \\
\hline \multicolumn{7}{|l|}{ Total dietary fat $(\mathrm{g} / \mathrm{d})$} \\
\hline First quartile ( $n$ 332) & 1.0 & Reference & 1.0 & Reference & 1.0 & Reference \\
\hline Second quartile ( $n 340$ ) & 0.74 & $0.51,1.09$ & 0.80 & $0.49,1.29$ & 0.69 & $0.41,1.15$ \\
\hline Third quartile ( $n 326$ ) & 0.83 & $0.57,1.21$ & 0.86 & $0.53,1.40$ & 0.81 & $0.48,1.34$ \\
\hline Fourth quartile $(n 333)$ & 0.83 & $0.57,1.20$ & 1.01 & $0.64,1.60$ & 0.65 & $0.39,1.10$ \\
\hline$P$ for trend & \multicolumn{2}{|r|}{0.41} & \multicolumn{2}{|r|}{0.86} & \multicolumn{2}{|r|}{0.16} \\
\hline \multicolumn{7}{|l|}{ Saturated fats $(g / d)$} \\
\hline First quartile $(n 334)$ & 1.0 & Reference & 1.0 & Reference & 1.0 & Reference \\
\hline Second quartile ( $n 340$ ) & 0.79 & $0.53,1.16$ & 1.05 & $0.64,1.74$ & 0.55 & $0.32,0.95$ \\
\hline Third quartile ( $n 326)$ & 0.99 & $0.68,1.46$ & $1 \cdot 17$ & $0.71,1.93$ & 0.85 & $0.51,1.42$ \\
\hline Fourth quartile $(n 331)$ & $1 \cdot 18$ & $0.81,1.72$ & 1.57 & $0.97,2.53$ & 0.90 & $0.54,1.50$ \\
\hline$P$ for trend & \multicolumn{2}{|r|}{0.24} & \multirow{2}{*}{\multicolumn{2}{|c|}{0.05}} & \multicolumn{2}{|r|}{1.00} \\
\hline \multicolumn{6}{|l|}{ Monounsaturated fats $(\mathrm{g} / \mathrm{d})$} & \\
\hline First quartile ( $n$ 335) & 1.0 & Reference & 1.0 & Reference & $1 \cdot 0$ & Reference \\
\hline Second quartile ( $n$ 334) & 0.91 & $0.63,1.32$ & 0.93 & $0.58,1.49$ & 0.91 & $0.55,1.49$ \\
\hline Third quartile ( $n$ 331) & 0.61 & $0.41,0.91$ & 0.68 & $0.41,1.20$ & 0.55 & $0.32,0.94$ \\
\hline Fourth quartile $(n 331)$ & 0.85 & $0.59,1.23$ & 0.97 & $0.62,1.53$ & 0.71 & $0.42,1.17$ \\
\hline$P$ for trend & \multicolumn{2}{|c|}{0.20} & \multicolumn{2}{|r|}{0.72} & \multicolumn{2}{|r|}{0.08} \\
\hline \multicolumn{7}{|l|}{ Total $n-3$ PUFA (g/d) } \\
\hline First quartile ( $n 316)$ & 1.0 & Reference & 1.0 & Reference & 1.0 & Reference \\
\hline Second quartile ( $n$ 336) & 0.90 & $0.61,1.32$ & 0.89 & $0.54,1.46$ & 0.92 & $0.55,1.54$ \\
\hline Third quartile $(n 344)$ & $1 \cdot 12$ & $0.77,1.64$ & 1.43 & $0.90,2 \cdot 28$ & 0.80 & $0.47,1.38$ \\
\hline Fourth quartile ( $n 335)$ & 0.79 & $0.53,1.17$ & 0.75 & $0.45,1.25$ & 0.82 & $0.48,1.39$ \\
\hline$P$ for trend & \multicolumn{2}{|r|}{0.32} & \multicolumn{2}{|r|}{0.41} & \multicolumn{2}{|r|}{0.43} \\
\hline \multicolumn{7}{|l|}{ Total $n-6$ PUFA (g/d) } \\
\hline First quartile ( $n$ 314) & 1.0 & Reference & $1 \cdot 0$ & Reference & $1 \cdot 0$ & Reference \\
\hline Second quartile ( $n$ 337) & 0.79 & $0.54,1.16$ & 0.73 & $0.45,1.17$ & 0.89 & $0.52,1.52$ \\
\hline Third quartile ( $n 340$ ) & 0.91 & $0.62,1.32$ & 0.79 & $0.49,1.26$ & 1.13 & $0.67,1.91$ \\
\hline Fourth quartile $(n 340)$ & 0.66 & $0.44,0.97$ & 0.64 & $0.40,1.03$ & \multirow{2}{*}{\multicolumn{2}{|c|}{0.20}} \\
\hline$P$ for trend & \multicolumn{2}{|r|}{0.06} & \multicolumn{2}{|r|}{0.11} & & \\
\hline \multicolumn{7}{|l|}{ Dietary cholesterol (mg/d) } \\
\hline First quartile ( $n 342)$ & 1.0 & Reference & 1.0 & Reference & 1.0 & Reference \\
\hline Second quartile ( $n 330)$ & 1.05 & $0.73,1.52$ & 1.25 & $0.78,2.01$ & 0.86 & $0.53,1.41$ \\
\hline Third quartile ( $n$ 335) & 0.85 & $0.58,1.25$ & 1.04 & $0.64,1.71$ & 0.70 & $0.42,1.18$ \\
\hline Fourth quartile $(n 324)$ & 0.89 & $0.61,1.30$ & 1.24 & $0.76,2 \cdot 00$ & 0.62 & $0.36,1.05$ \\
\hline$P$ for trend & & 0.39 & & 0.53 & & 0.06 \\
\hline
\end{tabular}

${ }^{*}$ Adjusted for age, sex, energy intake, BMI, smoking, hypertension and history of diabetes. 
Table 3. Association between food groups and prevalence of olfactory loss in the Blue Mountains Eye Study (Adjusted odds ratios and $95 \%$ confidence intervals, $n$ 1331)

\begin{tabular}{|c|c|c|c|c|c|c|}
\hline \multirow[b]{3}{*}{ Variable } & \multicolumn{6}{|c|}{ Severity of olfactory impairment ${ }^{\star}$} \\
\hline & \multicolumn{2}{|r|}{ Any } & \multicolumn{2}{|c|}{ Mild } & \multicolumn{2}{|c|}{ Moderate/severe } \\
\hline & OR & $95 \% \mathrm{Cl}$ & OR & $95 \% \mathrm{Cl}$ & OR & $95 \% \mathrm{Cl}$ \\
\hline \multicolumn{7}{|l|}{ Nuts (g/d) } \\
\hline First quartile ( $n$ 302) & 1.0 & Reference & 1.0 & Reference & 1.0 & Reference \\
\hline Second quartile ( $n 367$ ) & 0.65 & $0.45,0.94$ & 0.66 & $0.43,1.03$ & 0.64 & $0.38,1.08$ \\
\hline Third quartile ( $n 328$ ) & 0.59 & $0.40,0.87$ & 0.50 & $0.31,0.81$ & 0.67 & $0.40,1.14$ \\
\hline Fourth quartile ( $n$ 334) & 0.54 & $0.37,0.80$ & 0.42 & $0.25,0.68$ & 0.73 & $0.43,1.22$ \\
\hline$P$ for trend & \multirow{2}{*}{\multicolumn{2}{|c|}{0.01}} & \multicolumn{2}{|c|}{0.001} & \multicolumn{2}{|r|}{0.57} \\
\hline \multicolumn{5}{|l|}{ Margarine (g/d) } & & \\
\hline First quartile ( $n$ 327) & $1 \cdot 0$ & Reference & $1 \cdot 0$ & Reference & $1 \cdot 0$ & Reference \\
\hline Second quartile ( $n 266$ ) & 0.85 & $0.56,1.30$ & 1.05 & $0.61,1.81$ & 0.66 & $0.37,1.16$ \\
\hline Third quartile ( $n$ 531) & 1.03 & $0.72,1.46$ & $1 \cdot 24$ & $0.79,1.96$ & 0.85 & $0.53,1.35$ \\
\hline Fourth quartile (n 207) & 0.75 & $0.48,1.17$ & $1 \cdot 18$ & $0.68,2.05$ & 0.35 & $0.18,0.71$ \\
\hline$P$ for trend & \multicolumn{2}{|c|}{0.41} & \multicolumn{2}{|c|}{0.43} & \multicolumn{2}{|r|}{0.02} \\
\hline \multicolumn{7}{|l|}{ Butter (g/d) } \\
\hline First tertile $(n 587)$ & 1.0 & Reference & 1.0 & Reference & 1.0 & Reference \\
\hline Second tertile ( $n$ 409) & 0.74 & $0.54,1.02$ & 0.94 & $0.63,1.38$ & 0.51 & $0.32,0.81$ \\
\hline Third tertile ( $n$ 335) & 0.80 & $0.57,1.12$ & 0.78 & $0.50,1 \cdot 20$ & 0.84 & $0.53,1.32$ \\
\hline$P$ for trend & \multicolumn{2}{|c|}{0.46} & \multicolumn{2}{|c|}{0.27} & \multicolumn{2}{|r|}{0.83} \\
\hline \multicolumn{7}{|l|}{ Fish $(g / d)$} \\
\hline First quartile ( $n 334$ ) & 1.0 & Reference & 1.0 & Reference & 1.0 & Reference \\
\hline Second quartile ( $n$ 324) & 0.87 & $0.60,1.27$ & 0.69 & $0.43,1.10$ & $1 \cdot 18$ & $0.71,1.97$ \\
\hline Third quartile ( $n 336$ ) & 0.79 & $0.55,1.15$ & 0.81 & $0.52,1.26$ & 0.76 & $0.44,1.31$ \\
\hline Fourth quartile ( $n 337$ ) & 0.65 & $0.44,0.96$ & 0.50 & $0.31,0.82$ & 0.86 & $0.51,1.45$ \\
\hline$P$ for trend & \multicolumn{2}{|c|}{0.03} & \multicolumn{2}{|c|}{0.01} & \multicolumn{2}{|r|}{0.36} \\
\hline
\end{tabular}

*Adjusted for age, sex, energy intake, BMI, smoking, hypertension and history of diabetes.

$0.61(95 \%$ CI $0.37,1.00)$. Significant associations were not observed with other food groups (Table 4) or dietary fats (data not shown) and incidence of olfactory impairment.

\section{Discussion}

This cohort study provides novel epidemiological evidence of an inverse cross-sectional association between nut and fish consumption and any or mild olfactory impairment in older adults, independent of the effects of potential confounders such as age, smoking, BMI, energy intake and a history of diabetes and hypertension. In cross-sectional analyses, study participants who had the highest $v$. lowest intake of total n-6 PUFA and margarine had significantly reduced odds of olfactory loss. Significant associations were not observed with any of the key nutritional variables and the 5-year incidence of olfactory impairment.

Our findings of a non-significant association between dietary intakes of total dietary fats with both prevalence and 5-year incidence of olfactory impairment contrasts with the findings of a previous animal study, which showed that mice fed a high-fat diet showed a marked reduction in olfactory discrimination ${ }^{(9)}$. One potential reason for this divergent finding could be the type of test used to determine olfaction; in the present study we used a test that involved odour identification, while the animal study involved olfactory discrimination. We did, however, observe a marginally significant association between increasing dietary intakes of saturated fats and increasing odds of having prevalent mild olfactory loss, but not incident olfactory loss. Previously, it was shown that adoption of a saturated fat-enriched diet over a 3-week period was associated with an impairment of flow-mediated dilation, suggestive of endothelial dysfunction ${ }^{(29)}$. Given that endothelial dysfunction could contribute to the age-related decline in olfactory sensitivity observed in older adults ${ }^{(30)}$, increased saturated fat intake via endothelial dysfunction could be responsible for increasing the likelihood of having impaired olfaction.

Increasing nut consumption was independently associated with a reduced likelihood of having prevalent olfactory loss. Further, a marginally significant association was observed between increasing nut consumption at baseline and reduced risk of developing any olfactory impairment 5 years later. Given that the present study is a correlational study, it is not possible to establish the mechanisms that underlie the protective influence of nut consumption on olfactory function. However, we hypothesise that nuts are rich in unsaturated fats and other nutrients, which could decrease levels of inflammatory markers ${ }^{(31)}$ and, hence, help in maintaining healthy olfaction in older adults. This is because sensorineural olfactory loss is caused by damage or destruction of the neuroepithelium as a result of toxic inflammatory mediators and tissue disruption from infiltrating inflammatory cells ${ }^{(32,33)}$. In the BMES, we previously showed that nut consumption protected against dying from chronic inflammation-related diseases $^{(27)}$. Several bioactive components in nuts, acting in isolation or most probably synergistically, could explain the beneficial effect against inflammation ${ }^{(27,34)}$. It is likely that similar mechanisms could also mediate the association with olfactory impairment. For instance, $\mathrm{Mg}$, a mineral found in

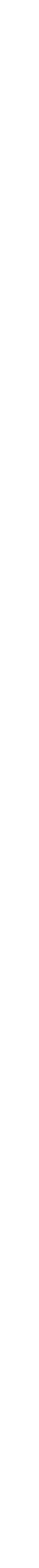


Table 4. Association between food groups and 5-year incidence of olfactory loss in the Blue Mountains Eye Study (Adjusted odds ratios and $95 \%$ confidence intervals, $n$ 667)

\begin{tabular}{|c|c|c|c|c|c|c|}
\hline \multirow[b]{3}{*}{ Variable } & \multicolumn{6}{|c|}{ Severity of olfactory impairment ${ }^{*}$} \\
\hline & \multicolumn{2}{|c|}{ Any } & \multicolumn{2}{|c|}{ Mild } & \multicolumn{2}{|c|}{ Moderate/severe } \\
\hline & OR & $95 \% \mathrm{Cl}$ & OR & $95 \% \mathrm{Cl}$ & OR & $95 \% \mathrm{Cl}$ \\
\hline \multicolumn{7}{|l|}{ Nuts (g/d) } \\
\hline First quartile ( $n$ 176) & 1.0 & Reference & 1.0 & Reference & 1.0 & Reference \\
\hline Second quartile ( $n$ 145) & 0.71 & $0.43,1.19$ & 0.72 & $0.40,1.29$ & 0.63 & $0.27,1.45$ \\
\hline Third quartile ( $n$ 175) & 0.70 & $0.43,1.15$ & 0.73 & $0.42,1.27$ & 0.70 & $0.31,1.59$ \\
\hline Fourth quartile ( $n$ 171) & 0.61 & $0.37,1.00$ & 0.62 & $0.35,1.09$ & 0.62 & $0.28,1.37$ \\
\hline$P$ for trend & \multicolumn{2}{|c|}{$0 \cdot 10$} & \multicolumn{2}{|c|}{0.17} & \multicolumn{2}{|r|}{0.39} \\
\hline \multicolumn{7}{|l|}{ Margarine $(g / d)$} \\
\hline First quartile ( $n$ 167) & $1 \cdot 0$ & Reference & 1.0 & Reference & 1.0 & Reference \\
\hline Second quartile ( $n$ 146) & 0.74 & $0.43,1.29$ & 0.71 & $0.39,1.31$ & 0.85 & $0.31,2.28$ \\
\hline Third quartile ( $n$ 246) & 0.86 & $0.53,1.38$ & 0.70 & $0.41,1 \cdot 21$ & 1.28 & $0.55,2.99$ \\
\hline Fourth quartile $(n+108)$ & 0.93 & $0.52,1.66$ & 1.00 & $0.54,1.88$ & 0.61 & $0.20,1.87$ \\
\hline$P$ for trend & \multicolumn{2}{|c|}{0.94} & \multicolumn{2}{|c|}{0.75} & \multicolumn{2}{|r|}{0.93} \\
\hline \multicolumn{7}{|l|}{ Butter (g/d) } \\
\hline First tertile $(n 287)$ & 1.0 & Reference & 1.0 & Reference & 1.0 & Reference \\
\hline Second tertile $(n 212)$ & $1 \cdot 28$ & $0.84,1.96$ & 1.47 & $0.90,2.39$ & 0.88 & $0.43,1.79$ \\
\hline Third tertile $(n 168)$ & 1.22 & $0.77,1.93$ & 1.33 & $0.78,2.25$ & 1.22 & $0.58,2.57$ \\
\hline$P$ for trend & \multicolumn{2}{|c|}{0.63} & \multicolumn{2}{|c|}{0.61} & \multicolumn{2}{|r|}{0.50} \\
\hline \multicolumn{7}{|l|}{ Fish $(g / d)$} \\
\hline First quartile ( $n$ 168) & 1.0 & Reference & 1.0 & Reference & 1.0 & Reference \\
\hline Second quartile ( $n$ 165) & 0.79 & $0.47,1.33$ & 0.74 & $0.41,1.33$ & 0.81 & $0.34,1.93$ \\
\hline Third quartile ( $n$ 168) & 0.79 & $0.47,1.32$ & 0.80 & $0.45,1.43$ & 0.72 & $0.30,1.73$ \\
\hline Fourth quartile ( $n$ 166) & 1.23 & $0.75,2.02$ & $1 \cdot 13$ & $0.64,1.98$ & 1.46 & $0 \cdot 66,3 \cdot 27$ \\
\hline$P$ for trend & \multicolumn{2}{|c|}{0.21} & \multicolumn{2}{|c|}{0.41} & \multicolumn{2}{|r|}{0.20} \\
\hline
\end{tabular}

*Adjusted for age, sex, energy intake, BMI, smoking, hypertension and history of diabetes.

nuts at higher amounts than in other edible plants ${ }^{(35)}$, could contribute to the reduction of inflammatory markers ${ }^{(34)}$. Moreover, dietary polyphenols present in nuts may have anti-inflammatory effects, mediated by both their antioxidant action and modulation of signal transduction pathways ${ }^{(27,34)}$. Finally, nuts also contain PUFA, including $\alpha$-linolenic acid and linoleic acid. $\alpha$-Linolenic acid has been shown to exert favourable effects on cytokine production ${ }^{(27,36)}$. This notion aligns with a previously hypothesised sensorineural mechanism in which olfactory dysfunction can occur with an intact neuroepithelium, as a consequence of direct interactions between olfactory sensory neurons and inflammatory cytokines ${ }^{(37)}$.

Moreover, inflammation could also be a step in the pathway that links fish consumption and reduced prevalence of any and mild olfactory impairment observed in the BMES. This is because fish contain $n-3$ PUFA (particularly EPA and DHA), which are demonstrated to regulate inflammatory processes and responses ${ }^{(38)}$. Alternatively, we speculate that olfactory loss could be a surrogate marker of neurodegeneration ${ }^{(39,40)}$ that could be accompanied by lower nut and fish consumption. There is growing evidence from both animal and human studies showing that moderate-duration dietary supplementation with nuts is capable of altering cognitive performance in human subjects, perhaps forestalling or reversing the effects of neurodegeneration in ageing ${ }^{(41)}$. Fish consumption could also confer a similar protective effect against neurodegeneration. For instance, there is epidemiological evidence showing that increased fish intake is associated with reduced Alzheimer's disease and reduced cognitive decline $^{(42)}$. Surprisingly, total and long-chain n-3 PUFA intake were not independently associated with the prevalence or incidence of olfactory loss in the present study. Evidence of the clinical benefits of long-chain $n-3$ PUFA, for example, is strong in some settings (e.g. rheumatoid arthritis) and yet weak in others (e.g. in asthma and inflammatory bowel disease $)^{(27,38)}$. The reasons for these apparently divergent findings are not clear, and warrant confirmation by other large, population-based cohorts.

In cross-sectional analyses, margarine and total n-6 PUFA consumption were associated with reduced odds of olfactory dysfunction. Given that margarine is one of the most convenient and readily available sources of linoleic acid (the main dietary $n$-6 PUFA), it was not surprising to see similar inverse associations between $n$ - 6 PUFA intake and margarine consumption and prevalence of olfactory impairment. There is still some uncertainty regarding the benefits and risks of $n$-6 PUFA intake ${ }^{(43,44)} \cdot n$-6 PUFA have long been considered as pro-inflammatory molecules because they are the main precursors of eicosanoids, a family of mediator molecules that are involved in immune and inflammatory responses ${ }^{(43,45)}$. However, in human subjects, higher intakes of n-6 fatty acids do not appear to be associated with elevated levels of inflammatory markers ${ }^{(43,46)}$. Therefore, the pathways mediating the relationship between $n-6$ PUFA and margarine intake and olfactory loss currently remains unclear. We caution, however, that this could be a chance finding, particularly as we did not see this association persist at the 5-year followup. Additional longitudinal studies are required to confirm or refute our observed associations with olfactory impairment. 
Strengths of the present study include its representative population-based sample with relatively high participation, longitudinal study design, availability of rich covariate/ confounder information, and the use of standardised objective measures of olfaction and the use of a validated FFQ to collect dietary data. The ability to classify participants with mild or moderate/severe olfactory loss also added value to this cohort study ${ }^{(1)}$. However, there are some caveats, such as dietary assessment by FFQ in which respondents have to estimate typical intake frequencies of food items and their portion sizes, which can potentially introduce measurement error and bias. However, the overall validity of fatty acid categories compared to weighted food records was moderately $\operatorname{good}^{(22,23,26)}$. In addition, the number of odorants in the SDOIT may limit the ability to detect small decrements in odour identification or to distinguish hyposmia from anosmia $^{(1,47)}$, and due to the nature of this test we cannot firmly establish whether an individual has olfactory dysfunction or not. Further, while the SDOIT has been validated in a US population, it has yet to be validated in an Australian population. Furthermore, persons with olfactory impairment compared to those with normal olfaction were shown to have lower survival ${ }^{(15)}$, and also given the older age of our cohort, many study participants were unable to attend or complete the follow-up examination. These factors could have contributed to the substantial reduction in participant numbers at 5-year follow-up. Hence, we had a relatively small number of incident cases of any olfactory loss over the 5 years, which could have led to insufficient power to detect modest associations between intakes of dietary fats and related food groups and olfactory loss 5 years later. We also need to highlight that the duration of follow-up is short and that the average age of the cohort is relatively old, perhaps most of the protective or beneficial effects from intakes of nuts, total n-6 PUFA and margarine, and so on, may have already been exerted before the cohort began to be followed. Hence, this could explain the significant association with prevalent olfactory loss at baseline but the lack of association with the 5-year incidence of olfactory loss. Finally, we cannot exclude the possibility of residual confounding, although we have attempted to adjust for a number of potential confounders, there could be many unmeasured or unaccounted variables (e.g. lifestyle and behavioural factors) that are likely to have influenced the overall pattern of food intake.

In summary, we show that increased consumption of total n-6 PUFA, margarine, nuts, and fish could contribute to a modest reduction in the prevalence of olfactory impairment, independent of the potential confounding influences of age, sex, smoking, BMI and a history of hypertension and diabetes. Apart from a marginally significant inverse association between nut consumption and 5-year incidence of olfactory impairment, dietary intakes of fatty acids or related food groups did not influence risk of incident olfactory loss. The reasons for these observed associations are not clear and require confirmation in other large population-based studies. These further studies will also be useful in establishing whether consumption of nuts and fish might have a role in the prevention of olfactory dysfunction in older adults.

\section{Acknowledgements}

The Blue Mountains Eye and Hearing Studies were supported by the Australian National Health and Medical Research Council (grant nos. 974159, 991407, 211069, 262120).

The authors' contributions are as follows: B. G. and P. M. were involved in the study concept and design; P. M. was responsible for the acquisition of data; B. G., C. M. S., V. M. F. and P. M. were involved in the interpretation of data; B. G. was involved in the drafting of the manuscript; G. B. performed the analyses of data; B. G., C. M. S., V. M. F. and P. M. were involved in critical revision of the manuscript.

All authors have no conflict of interest and declare no financial interest.

\section{References}

1. Gopinath B, Anstey KJ, Kifley A, et al. (2012) Olfactory impairment is associated with functional disability and reduced independence among older adults. Maturitas $\mathbf{7 2}$, $50-55$.

2. Karpa MJ, Gopinath B, Rochtchina E, et al. (2010) Prevalence and neurodegenerative or other associations with olfactory impairment in an older community. J Aging Health 22, 154-168.

3. Murphy C, Schubert CR, Cruickshanks KJ, et al. (2002) Prevalence of olfactory impairment in older adults. JAMA $\mathbf{2 8 8}$, 2307-2312.

4. Doty RL (2009) The olfactory system and its disorders. Semin Neurol 29, 074-081.

5. Kalmey JK, Thewissen JGM \& Dluzen DE (1998) Age-related size reduction of foramina in the cribriform plate. Anat Rec 251, 326-329.

6. Doty RL, Shaman P, Applebaum SL, et al. (1984) Smell identification ability: changes with age. Science (New York, NY) 226, 1441-1443.

7. Doty RL, McKeown DA, Lee WW, et al. (1995) A study of the test-retest reliability of ten olfactory tests. Chem Senses 20, 645-656.

8. Boyce JM \& Shone GR (2006) Effects of ageing on smell and taste. Postgrad Med J 82, 239-241.

9. Miwa T, Furukawa M, Tsukatani T, et al. (2001) Impact of olfactory impairment on quality of life and disability. Arch Otolaryngol Head Neck Surg 127, 497-503.

10. Smeets MAM, Veldhuizen MG, Galle S, et al. (2009) Sense of smell disorder and health-related quality of life. Rehabil Psychol 54, 404-412.

11. Gopinath B, Anstey KJ, Sue CM, et al. (2011) Olfactory impairment in older adults is associated with depressive symptoms and poorer quality of life scores. Am J Geriatr Psychiatry 19, 830-834.

12. Pollatos O, Albrecht J, Kopietz R, et al. (2007) Reduced olfactory sensitivity in subjects with depressive symptoms. J Affect Disord 102, 101-108.

13. Nordin S, Monsch AU \& Murphy C (1995) Unawareness of smell loss in normal aging and Alzheimer's disease: discrepancy between self-reported and diagnosed smell sensitivity. J Gerontol B Psychol Sci Soc Sci 50, P187-P192.

14. Ross GW, Petrovitch H, Abbott RD, et al. (2008) Association of olfactory dysfunction with risk for future Parkinson's disease. Ann Neurol 63, 167-173.

15. Gopinath B, Sue CM, Kifley A, et al. (2012) The association between olfactory impairment and total mortality in older adults. J Gerontol A Biol Sci Med Sci 67A, 204-209. 
16. Thiebaud N, Johnson MC, Butler JL, et al. (2014) Hyperlipidemic diet causes loss of olfactory sensory neurons, reduces olfactory discrimination, and disrupts odor-reversal learning. J Neurosci 34, 6970-6984.

17. Attebo K, Mitchell P \& Smith W (1996) Visual acuity and the causes of visual loss in Australia. The Blue Mountains Eye Study. Ophthalmology 103, 357-364.

18. Morgan CD, Nordin S \& Murphy C (1995) Odor identification as an early marker for Alzheimer's disease: impact of lexical functioning and detection sensitivity. J Clin Exp Neuropsychol 17, 793-803.

19. Doty RL (2007) Office procedures for quantitative assessment of olfactory function. Am J Rhinol 21, 460-473.

20. Ekman G, Berglund B, Berglund U, et al. (1967) Perceived intensity of odor as a function of time of adaptation. Scand J Psychol 8, 177-186.

21. Willett WC, Sampson L, Browne ML, et al. (1988) The use of a self-administered questionnaire to assess diet four years in the past. Am J Epidemiol 127, 188-199.

22. Smith W, Mitchell P, Reay EM, et al. (1998) Validity and reproducibility of a self-administered food frequency questionnaire in older people. Aust $N Z J$ Public Health 22, 456-463.

23. Lewis J, Hunt A \& Milligan G (1995) NUTTAB95 Nutrient Data Table for Use in Australia. Canberra: Australian Government Publishing Service.

24. Mann N, Pirotta Y, O'Connell S, et al. (2006) Fatty acid composition of habitual omnivore and vegetarian diets. Lipids 41, 637-646.

25. Flood VM, Webb KL, Rochtchina E, et al. (2007) Fatty acid intakes and food sources in a population of older Australians. Asia Pac J Clin Nutr 16, 322-330.

26. Gopinath B, Flood VM, Rochtchina E, et al. (2010) Consumption of $\omega-3$ fatty acids and fish and risk of age-related hearing loss. Am J Clin Nutr 92, 416-421.

27. Gopinath B, Buyken AE, Flood VM, et al. (2011) Consumption of polyunsaturated fatty acids, fish, and nuts and risk of inflammatory disease mortality. Am J Clin Nutr 93 1073-1079.

28. Whitworth JA, World Health Organization \& International Society of Hypertension Writing Group (2003) 2003 World Health Organization (WHO)/International Society of Hypertension (ISH) statement on management of hypertension. J Hypertens 21, 1983-1992.

29. Keogh JB, Grieger JA, Noakes M, et al. (2005) Flow-mediated dilatation is impaired by a high-saturated fat diet but not by a high-carbohydrate diet. Arterioscler Thromb Vasc Biol 25, $1274-1279$

30. Getchell ML, Shah DS, Buch SK, et al. (2003) 3-Nitrotyrosine immunoreactivity in olfactory receptor neurons of patients with Alzheimer's disease: implications for impaired odor sensitivity. Neurobiol Aging 24, 663-673.
31. Jiang R, Jacobs DR, Mayer-Davis E, et al. (2006) Nut and seed consumption and inflammatory markers in the multi-ethnic study of atherosclerosis. Am J Epidemiol 163, 222-231.

32. Kern RC (2000) Chronic sinusitis and anosmia: pathologic changes in the olfactory mucosa. Laryngoscope 110, 1071-1077.

33. Yee KK, Pribitkin EA, Cowart BJ, et al. (2010) Neuropathology of the olfactory mucosa in chronic rhinosinusitis. $A m \mathrm{~J}$ Rhinol Allergy 24, 110-120.

34. Ros E (2009) Nuts and novel biomarkers of cardiovascular disease. Am J Clin Nutr 89, 1649S-1656S.

35. Segura R, Javierre C, Lizarraga MA, et al. (2006) Other relevant components of nuts: phytosterols, folate and minerals. Br J Nutr 96, Suppl. 2, S36-S44.

36. Zhao G, Etherton TD, Martin KR, et al. (2007) Dietary $\alpha$-linolenic acid inhibits proinflammatory cytokine production by peripheral blood mononuclear cells in hypercholesterolemic subjects. Am J Clin Nutr 85, 385-391.

37. Sultan B, May LA \& Lane AP (2011) The role of TNF- $\alpha$ in inflammatory olfactory loss. The Laryngoscope $\mathbf{1 2 1}$ 2481-2486.

38. Calder PC (2006) n-3 Polyunsaturated fatty acids, inflammation, and inflammatory diseases. Am J Clin Nutr 83, S1505-1519S.

39. Mesholam RI, Moberg PJ, Mahr RN, et al. (1998) Olfaction in neurodegenerative disease: a meta-analysis of olfactory functioning in Alzheimer's and Parkinson's diseases. Arch Neurol 55, 84-90.

40. Seo H-S, Jeon K, Hummel T, et al. (2009) Influences of olfactory impairment on depression, cognitive performance, and quality of life in Korean elderly. Eur Arch Otorbinolaryngol 266, 1739-1745.

41. Pribis P \& Shukitt-Hale B (2014) Cognition: the new frontier for nuts and berries. Am J Clin Nutr 100, 347S-352S.

42. Cole GM, Ma Q-L \& Frautschy SA (2009) $\omega$-3 Fatty acids and dementia. Prostaglandins Leukot Essent Fatty Acids 81, 213-221.

43. Czernichow S, Thomas D \& Bruckert E (2010) n-6 Fatty acids and cardiovascular health: a review of the evidence for dietary intake recommendations. Br J Nutr 104, 788-796.

44. Ramsden CE, Zamora D, Leelarthaepin B, et al. (2013) Use of dietary linoleic acid for secondary prevention of coronary heart disease and death: evaluation of recovered data from the Sydney Diet Heart Study and updated meta-analysis. BMJ 346, e8707.

45. Calder PC (2001) Polyunsaturated fatty acids, inflammation, and immunity. Lipids 36, 1007-1024.

46. Ferrucci L, Cherubini A, Bandinelli S, et al. (2006) Relationship of plasma polyunsaturated fatty acids to circulating inflammatory markers. J Clin Endocrinol Metab 91, 439-446.

47. Schubert CR, Cruickshanks KJ, Fischer ME, et al. (2012) Olfactory impairment in an adult population: the Beaver Dam Offspring Study. Chem Senses 37, 325-334. 